\title{
Correction to: Implementation of Energy Efficient WBAN Using IEEE 802.15.6 Scheduled Access MAC with Fast DWT Based Backhaul Data Compression for e-Healthcare
}

Tanumay Manna and Iti Saha Misra

\begin{abstract}
Correction to:
Chapter "Implementation of Energy Efficient WBAN Using IEEE 802.15.6 Scheduled Access MAC with Fast DWT Based Backhaul Data Compression for e-Healthcare" in:

S. Biswas et al. (Eds.): Communication Systems and Networks, LNCS 11227, https://doi.org/10.1007/978-3-030-10659-1_2
\end{abstract}

The original version of this chapter was revised. Reference no. 23 ("References" section) was updated because the chapter which was under review has now been published. 